\title{
Dust emission from clusters of galaxies: statistical detection
}

\author{
L. A. Montier and M. Giard
}

\author{
Centre d'Études Spatiales des Rayonnements, 9 avenue Colonel Roche, 31022 Toulouse, France \\ e-mail: montier@cesr.fr
}

Received 18 November 2004 / Accepted 3 March 2005

\begin{abstract}
The detection of the IR emission from individual galaxy clusters is a difficult task due to the extremely low level of this emission and the fluctuations of the IR sky, galactic cirrus and background galaxies. We have statistically detected a significant IR emission toward galaxy clusters at $12 \mu \mathrm{m}, 25 \mu \mathrm{m}, 60 \mu \mathrm{m}$ and $100 \mu \mathrm{m}$ by co-adding the IRAS maps toward a total of 11507 galaxy clusters extracted from the CDS database. This process averages the sky fluctuations of the astrophysical sky and the survey noise to a very low level in all four bands. We have obtained an averaged central detection at all wavelengths, with an intensity of $2685 \pm 573 \mathrm{Jy} / \mathrm{sr}, 2127 \pm 742 \mathrm{Jy} / \mathrm{sr}, 16253 \pm 670 \mathrm{Jy} / \mathrm{sr}$ and $34011 \pm 1391 \mathrm{Jy} / \mathrm{sr}$ respectively. These detections have been confirmed against possible spurious or systematic signals using different test methods. The origin of this detected IR emission is discussed. The free-free contribution to the IR emission can be ruled out. Both the level of the emission and its spectrum favor an interpretation in terms of dust emission. However the exact location of this dust remains unknown: is this emission due to intergalactic dust, or simply the dust included in the galaxies of the clusters? This last question will be discussed in a forthcoming paper.
\end{abstract}

Key words. intergalactic medium - galaxies: clusters: general - infrared: stars

\section{Introduction}

The presence of dust in the intracluster medium (hereafter ICM) is an old but still open question. On the one hand Zwicky et al. (1957) and Karachentsev \& Lipovetskii (1969) were among the first to study this problem when looking at the extinction of very far clusters of galaxies by rich and nearby clusters. Bogart \& Wagoner (1973) have performed similar observations during quasar counts (see also Boyle et al. 1988; Romani \& Maoz 1992), but there was no clear evidence of such intergalactic dust absorption. On the other hand the collisionnal heating of grains in hot plasmas has been proposed by Dwek (1987) as an emission mechanism to power the FIR emission from remnants supernovae, and recently extended to the FIR emission from intergalactic dust. Such detections of dust emission, based on the IRAS data, have been performed in the intracluster medium with an uneven success. Hu (1992) estimated a mean surface brightness of $46650 \pm 16550 \mathrm{Jy} / \mathrm{sr}$ at $100 \mu \mathrm{m}$ on a small sample of 9 galaxy clusters. The dust emission from cD galaxies inside galaxy clusters has been stressed by Cox et al. (1995) and Bregman et al. (1990) for 10\% to 30\% (respectively) of the objects studied. Wise et al. (1993) tried to detect the extended IR emission of 56 galaxy clusters, but without success: only five clusters were detected, but with a low level of significance. More recently Stickel et al. $(1998,2002)$ used ISO data and detected a colour excess in the surface brightness ratio $I_{120 \mu \mathrm{m}} / I_{185 \mu \mathrm{m}}$ toward the Coma Cluster. They derived a $120 \mu \mathrm{m}$ flux excess of $\approx 200000 \mathrm{Jy} / \mathrm{sr}$, interpreted as thermal emission from intracluster dust. However this interpretation is still very debated. Quillen et al. (1999) attributed this emission to the integrated emission of the inner galaxies of the cluster. The presence or absence of dust in the ICM is crucial to understanding the physics of the galaxy clusters. Indeed the dust can be considered an efficient cooling agent of the ICM at a large scale (as efficient as the cooling by X-ray emission) and a heating agent at small scales (see Montier \& Giard 2004).

From the theoretical point of view, the dust pollution of the intergalactic medium (hereafter IGM) can be explained by several processes (see Aguirre et al. 2001a,c; Gnedin \& Ostriker 1997; Cen \& Ostriker 1999; Duc et al. 2002). Cosmological simulations have been performed to estimate the amount of dust in the IGM (Aguirre et al. 2001b), leading to a dust-togas mass ratio of $Z_{\mathrm{d}}=2 \times 10^{-5}$. Moreover some evidence of an intergalactic stellar population has been pointed out (see Feldmeier et al. 1998; Theuns \& Waren 1997; Durrell et al. 2002; Ferguson et al. 1998; Gal Yam et al. 2003; Gerhard et al. 2002), opening the possibility of an in situ dust enrichment of the ICM. However the predictions of detectability in the FIR spectrum derived by Popescu et al. (2000) for grains produced by the intergalactic stellar population in the Virgo Cluster yield a level of dust emission close to $10000 \mathrm{Jy} / \mathrm{sr}$ at $100 \mu \mathrm{m}$, which is 10 times lower than the IRAS sensitivity. Moreover, the statistical fluctuation of the infrared sky is a strong limitation. On the one hand the fluctuation of galactic cirrus in the IRAS maps leads to a noise confusion level of about $1.3 \mathrm{MJy} / \mathrm{sr}$ at $100 \mu \mathrm{m}$ (see Gautier et al. 1992; Helou \& Beichman 1990). 
On the other hand the Cosmic Far-InfraRed Background, due to the accumulated light of extragalactic unresolved galaxies (see Guiderdoni et al. 1997), yields a noise confusion level lower than $1.1 \mathrm{MJy} / \mathrm{sr}$ at $90 \mu \mathrm{m}$ (see Kiss et al. 2001).

Neverthless we have used a statistical method, first introduced by Kelly \& Rieke (1990) to obtain an estimate of the average dust emission from clusters of galaxies, instead of dealing with only one cluster. They stacked IRAS scans centered on 71 galaxy clusters at 60 and $100 \mu \mathrm{m}$ to increase the cluster signal and smooth the foreground signal. Their aim was to study the evolution of the galactic emission, using the galaxy clusters as indicators of galaxies at high redshift. They derived an average intensity of the galaxy clusters of $34600 \pm 6200 \mathrm{Jy} / \mathrm{sr}$ at $60 \mu \mathrm{m}$, and an upper limit of $36700 \mathrm{Jy} / \mathrm{sr}$ at $100 \mu \mathrm{m}$. This emission was only modeled by the galactic component of the clusters, without taking into account a possible intergalactic component. In our work, we have used the same method, but applied on maps instead of scans. This provided a better removal of the foreground fluctuation due to the spherical symmetry. Because of the huge increase in the number of galaxy clusters averaged, we have extended our study to the shorter wavelengths 12 and $25 \mu \mathrm{m}$.

This work is divided in two papers. In this first paper (Paper I), the stacking method is described in detail (see Sect. 2), and our first estimates of the dust IR emission from galaxy clusters are presented (see Sect. 3). An extensive analysis and modelling of these detections will be performed in the second part of this work (Paper II).

\section{The "stacking" method}

The dust emission from a single galaxy cluster is rarely detectable because of the level of the IR radiation background emited by the Milky Way and infrared galaxies (see Sect. 1). In order to decrease the contribution of these components and the initial noise level of the data, we add the hypothetical central emission of several galaxy clusters and smooth the background fluctuation emission by averaging local maps centered on the galaxy clusters. In this section, we will detail the different steps of this 'stacking method', from the extraction of the clusters maps to the point source removal and finally to the cluster sample selection.

\subsection{Extraction of the galaxy clusters maps}

The local maps of the clusters of galaxies have been extracted from the new generation of IRAS maps: IRIS (Improved Reprocessing of the IRAS Survey, Miville-Deschênes \& Lagache 2004). Like the previous generation of IRAS maps, the IRIS maps consist of a mosaic of 430 tangent plates covering the full sky with pixels of 1.5 arcmin, at the four IRAS wavelengths $12,25,60$ and $100 \mu \mathrm{m}$. These new maps have been corrected for the initial defects of the previous ISSA catalogue (IRAS Sky Survey Atlas, described in detail in the IRAS Sky Survey Atlas Explanatory Supplement, Wheelock et al. 1994), such as striping, calibration, zero level and zodiacal light. Whereas the emission from cold grains spans from a few $\mu \mathrm{m}$ to more than $2 \mathrm{~mm}$ and peaks between $100 \mu \mathrm{m}$ and
$500 \mu \mathrm{m}$ depending on the dust temperature, the IRAS wavelength range matches only the low wavelength part of the dust spectrum. Moreover, the angular size of galaxy clusters can be very small (an arcmin) or very high (a few degrees for the Coma Cluster for example). But for this study, we are interested in a very large sample of galaxy clusters, and the mean size is about a few arcmin. The IRIS resolutions are $3.8^{\prime} \pm 0.2^{\prime}$, $3.8^{\prime} \pm 0.2^{\prime}, 4.0^{\prime} \pm 0.2^{\prime}$ and $4.3^{\prime} \pm 0.2^{\prime}$ at respectively $12,25,60$ and $100 \mu \mathrm{m}$ (Miville-Deschênes \& Lagache 2004), which are in a good agreement with the angular size of the galaxy clusters mentioned above.

The choice of the galaxy clusters catalogue depends on the following factors: the coordinates, the size, the redshift, or the temperature and the total number of clusters in the catalogue. We will see that these different aspects are useful for the completeness of this work, requiring the use of different catalogues (see Paper II). In Paper I, the catalogue was obtained directly from the Simbad database (http://simbad.u-strasbg.fr/sim-fid.pl): it consists of 22344 references to clusters of galaxy with coordinates only. It has been used to optimize the signal-to-noise ratio, in order to constrain the confidence level of the detection, see Sect. 3 .

For each catalogue of galaxy clusters a set of local maps centered on each galaxy cluster has been extracted from the IRAS survey. These local cluster maps are square maps of $21 \times$ 21 pixels ( $992.25 \mathrm{arcmin}^{2}$ ). We thus obtain a set $C$ of $N_{\mathrm{C}}$ cluster maps $M$ of $N_{\text {Pix }}=441$ pixels:

$$
C=\left(\left(M_{i}\right)^{k}\right)
$$

for $i=1$ to $N_{\mathrm{Pix}}$, and $k=1$ to $N_{\mathrm{C}}$, at the wavelengths 12,25 , 60 and $100 \mu \mathrm{m}$.

\subsection{Selection of a homogeneous sample}

Before stacking the galaxy clusters maps at each wavelength, we have to be very careful about the homogeneity of the sample. We have seen that the cluster IR emission is undetectable on a single map, so we would like to decrease the background emission by using its random distribution from one map to the other. This implies that all the maps have the same contribution to the background. For this reason, we i) have to deal carefully with the point sources (Point source removal); ii) to level all the cluster maps around a mean intensity close to zero (Background removal) and iii) more generally to carefully chose the galaxy clusters maps included in the stack (Sample selection).

\subsubsection{Point source removal}

Point sources represent high disruptions for the stacking method. They locally exceed the emission of the rest of the stacked map, and so break the first assumption of homogeneity between pixels and between cluster maps. We have used the IRAS Point Source Catalogue (PSC), containing 245889 identified point sources (Beichman et al. 1988), to detect and remove them from the cluster maps at each wavelength. Given a cluster map $M^{k}$, if a point source of the PSC is detected inside the cluster map or near the border at one wavelength, 
a square of $5 \times 5$ pixels $\left(56.25 \mathrm{arcmin}^{2}\right)$ around the point source is flagged for all 4 wavelengths. A weighted map $W^{k}$ is then associated with the cluster map $M^{k}$, with the following definition: $W_{i}^{k}=0$ for flagged pixel $i, W_{i}^{k}=1$ otherwise. During the stacking process, all the flagged pixels will not be taken into account, as detailed in Sect. 2.3.

Moreover when a flagged pixel is detected inside a square of $3 \times 3$ pixels $\left(20.25 \mathrm{arcmin}^{2}\right)$ around the center of the cluster map, the whole map is removed from the stack. A vector "def" is introduced as follows: $\operatorname{def}_{k}=1$ if the cluster map $M^{k}$ is present in the final stack, and $\operatorname{def}_{k}=0$ otherwise. Thus in the case of point sources, we have: $\operatorname{def}_{k}=0$ if $W^{k}=0$ in a centered square of the cluster map. By this process of point sources removal, the homogeneity of the pixels inside a cluster map is checked, without decreasing the central signal by stacking cluster maps with no signal in the galaxy cluster area.

\subsubsection{Background removal}

We are only interested in the cluster emission excess at the center of the map. Thus we can substract for each map a "background" equal to the mean of the unflagged pixels of the cluster map. For each map $M^{k}$, the new map $\widetilde{M}^{k}$ is then given by:

$\widetilde{M}^{k}=M^{k}-\frac{\sum_{i} W_{i}^{k} \cdot M_{i}^{k}}{\sum_{i} W_{i}^{k}}$.

By this process, all the cluster maps have an average around zero. This limits the discrepancy from one cluster map to another, without perturbing the central cluster IR emission excess, if it exists. We stress that only the excess is unchanged, so it has to be calculated by comparison with the average of the background level, that is slightly negative.

\subsubsection{Sample selection}

The initial sample $C$ containing $N_{\mathrm{C}}$ galaxy clusters has already been reduced during the first step of this work, the point source removal. The vector "def", introduced in Sect. 2.2.1, dealing with the presence of a cluster map in the final stack, has already been updated there. The second selection phase consists of removing from the stack all the galaxy clusters that have a galactic latitude of less than 10 degrees.

The last step in the sample selection is based on the pixel standard deviation $\sigma_{k}$ inside each cluster map $\widetilde{M}^{k}$ :

$\sigma_{k}=\sqrt{\frac{\sum_{i}\left(W_{i}^{k} \cdot\left(\widetilde{M}_{i}^{k}\right)^{2}\right)}{\sum_{i} W_{i}^{k}} .}$

The rms of each map is then compared to the average rms of all maps remaining in the stack $\sigma_{\text {stack }}$ :

$\sigma_{\text {stack }}=\sqrt{\frac{\sum_{k}\left(\operatorname{def}_{k} \cdot\left(\sigma_{k}\right)^{2}\right)}{\sum_{k} \operatorname{def}_{k}}}$.

The cluster maps that have a $\sigma_{k}$ too large wrt $\sigma_{\text {stack }}$ are declared undefined. Thus we have for each cluster map $\bar{M}^{k}$ :

$\operatorname{def}_{k}=0$ if $\sigma_{k}>4 \sigma_{\text {stack}}$.
This removal process is repeated until no more cluster maps remain to be removed from the remaining stack. This is done for each wavelength and applied to the other wavelengths in order to keep the same sample for the four IRAS wavelengths. The sample of the remaining cluster maps is now considered sufficiently homogeneous to proceed to the final step of the method. The final sample $\widetilde{C}$ is defined:

$\widetilde{C}=\left(\left(\widetilde{M}_{i}\right)^{k}\right)$

for $k=1$ to $N_{\mathrm{C}}$ and $\operatorname{def}_{k}=1$, leading to $\widetilde{N}_{C}$ cluster maps.

\subsection{Stacking the selected maps}

We have obtained a new set $\widetilde{C}$ of $\widetilde{N}_{\mathrm{C}}$ cluster maps $\widetilde{M}^{k}$ and associated weighted maps $W^{k}$. This new sample has been chosen to be homogeneous by flagging the pixels associated with point sources inside each cluster map (see Sect. 2.2.1), and by removing from the stack all the cluster maps that have a too high variance compared to the rest of the sample (see Sect. 2.2.3). Moreover, in order to avoid systematic pollution by the largescale features of our galaxy, due to the orientation of the initial IRIS mosaic maps, the cluster maps are randomly rotated by 0 , 90, 180 or 270 degrees before summing. This tends to further homogenize the background component, and to increase the signal-to-noise ratio. The function $\operatorname{rot}_{k}$ is introduced, and returns for every $k$ the cluster map $M_{k}$ rotated by $0,90,180$ or 270 degrees. The final stacked map $S$ is then given for every pixel $i$ by:

$S_{i}=\frac{\sum_{k}\left(\operatorname{rot}_{k}\left(W_{i}^{k} \cdot \widetilde{M}_{i}^{k}\right)\right)}{\sum_{k} \operatorname{rot}_{k}\left(W_{i}^{k}\right)}$

for $k=1$ to $N_{\mathrm{C}}$ and $\operatorname{def}_{k}=1$.

This general process is performed simultaneously at the various wavelengths. This finally leads to a set of four stacked maps $S^{12}, S^{25}, S^{60}$ and $S^{100}$, at $12,25,60$ and $100 \mu \mathrm{m}$ respectively, obtained with stricly the same selection of pixels (point source removal) and cluster maps (sample selection).

\section{First detections and tests}

The stacking method described in Sect. 2 has been applied to the Simbad catalogue. We will present the IR emission observed at the center of the stacked map, and we will then confirm this detection by various tests.

\subsection{Description of the catalogue}

We have used the Simbad catalogue to increase as much as possile the signal-to-noise ratio, without taking into account the geometry of the galaxy clusters summed. This catalogue includes 22344 galaxy clusters coordinates, extracted from all the available catalogues. More than $90 \%$ of the galaxy clusters of the Simbad catalogue are taken from 14 catalogues, described in detail in Table 1. The largest are: the "Northern Sky Optical Cluster Survey" (NSC) (Gal et al. 2003) that includes more than 8000 objects in the northern hemisphere. 
Table 1. The 14 larger galaxy cluster catalogues included in the initial Simbad catalogue, and representing more than $90 \%$ of the intial entire catalogue. Second column: number of objects initially present in the catalogue. Third column: number of clusters in the reduced Simbad catalogue checked for overlaps.

\begin{tabular}{cccl}
\hline \hline $\begin{array}{c}\text { Catalogue } \\
\text { Name }\end{array}$ & \multicolumn{2}{c}{ Number of clusters } & Reference \\
initial & reduced & \\
\hline NSC & 8131 & 6202 & Gal et al. (2003) \\
ACO & 5203 & 4060 & Abell et al. (1989) \\
LCDCS & 1174 & 405 & Gonzales et al. (2001) \\
ZwCl & 787 & 564 & Zwicky et al. (1961) \\
APMCC & 787 & 529 & Dalton et al. (1997) \\
BMA2003 & 745 & 484 & Bahcall et al. (2003) \\
Str & 656 & 501 & Duus \& Newell (1977) \\
WBL & 488 & 415 & White et al. (1999) \\
PLO2002 & 444 & 31 & Postman et al. (2002) \\
EDCC & 394 & 330 & Lumsden et al. (1992) \\
S85 & 384 & 270 & Shectman (1985) \\
GHO & 380 & 193 & Gunn et al. (1986) \\
RXJ & 284 & 181 & Rosat X cat \\
EIS & 247 & 35 & Scodeggio et al. (1999) \\
\hline
\end{tabular}

The ABELL catalogue (Abell et al. 1989) compiles about 5000 references on the whole sky. The LCDCS for "Las Campanas Distant Cluster Survey" (Gonzales et al. 2001) focuses on galaxy clusters with high redshift $(z \geq 0.3)$. The APM Cluster Catalogue (Dalton et al. 1997) has been extracted from the APM Galaxy survey. The BMA2003 catalogue (Bahcall et al. 2003) is derived from the SDSS ("Sloan Digital Sky Survey") yielding a selection of small redshift objects $(0.05 \leq z \leq 0.3)$. The Zwicky cluster catalogue (Zwicky et al. 1961) is the oldest of the compilation. Because of this variety of catalogues, the Simbad catalogue contains galaxy clusters with various known properties, concerning redshift, density, or temperature.

Neverthless, this global catalogue has not been checked for overlaps, if not already identified in the literature. In order to avoid such duplications of cluster references, all the galaxy clusters closer than $15^{\prime}$ to another one have been removed from the initial catalogue, leading to the removal of 6836 references, or about $30 \%$ of the initial Simbad catalogue. Moreover 920 clusters located in undefined regions of the IRIS maps have been removed. This leads to a reduced Simbad catalogue of 14588 objects, described in detail in Table 1 for its 14 largest components. Some catalogues have almost entirely been excluded, like the [PLO2002] (Postman et al. 2002), or the EIS (Scodeggio et al. 1999). By this process the local maps extracted from the IRIS database contain only one galaxy cluster.

After the different selections of cluster maps described in Sect. 2.2, 78.8\% of the initial cluster maps have been kept, leading to a sample of 11507 cluster maps. On this final sample, only $2 \%$ of the pixels were flagged due to the presence of point sources. Figures 1 and 2 illustrate that the galaxy clusters removal from the initial cluster sample show the same sky distribution as the initial catalogue.

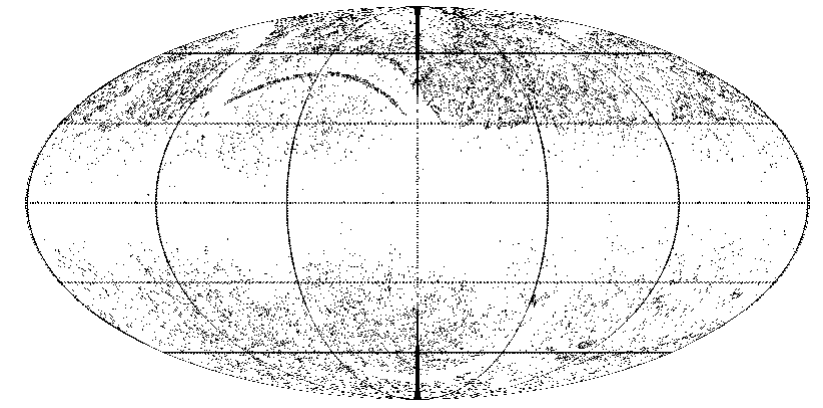

Fig. 1. Distribution of the galaxy clusters of the initial SIMBAD catalogue, in galactic J2000 coordinates.

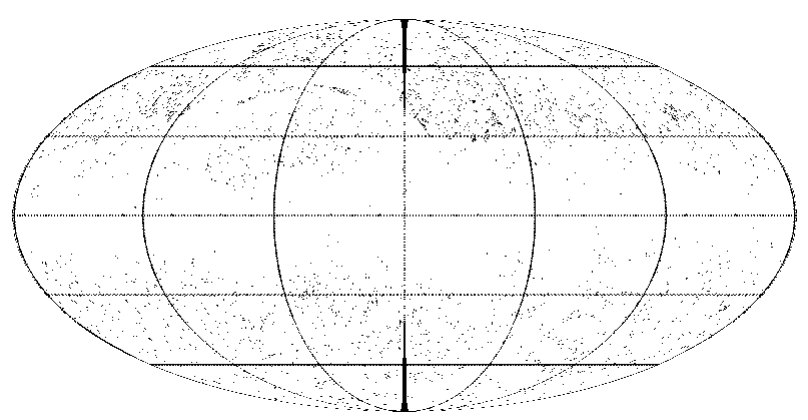

Fig. 2. Distribution of the galaxy clusters of the SIMBAD catalogue removed from the initial sample after the various selection steps of the stacking process, in galactic J2000 coordinates.

\subsection{Detection of the dust emission}

The gradual rise of the central peak at $100 \mu \mathrm{m}$ is illustrated in Fig. 3, at 6 intermediate steps of the stacking process: 10, 50, 200, 1000, 4000 and 11507 (all) galaxy cluster maps taken into account. The progressive smoothing of the background and the emergence of the central emission are obvious on these few pannels. The final stacked maps $S^{12}, S^{25}, S^{60}$ and $S^{100}$ (at 12, 25,60 and $100 \mu \mathrm{m}$ respectively) are displayed on the left pannels of Fig. 4. The dust emission peak is clearly observed at $12 \mu \mathrm{m}, 60 \mu \mathrm{m}$ and $100 \mu \mathrm{m}$, with a signal-to-noise ratio equal to $4.7,24.3$ and 24.5 respectively. The detection at $25 \mu \mathrm{m}$ is not as clear, with a signal-to-noise ratio just about 2.9. The intensity of the central peak of the average emission from clusters of galaxies (see Table 2) has been estimated as the difference between the central peak and the background level (defined as the average of the pixels beyond a radius of $7.5^{\prime}$ from the central peak) below zero, as already emphasized in Sect. 2.2.2. The $1 \sigma$ error has been derived from the rms of the background pixels (beyond a radius of 7.5') on the final map. These error bars can be compared with the upper limits $\sigma_{\text {IRIS }}$ of the noise level of the IRIS maps (Miville-Deschênes \& Lagache 2004) divided by $\sqrt{11507}$ (4th column of Table 2 ). We observe that $\sigma>\sigma_{\text {IRIS }}$ at all wavelengths, which confirms the validity of our error bars.

The intensity profiles (see Fig. 5) have been computed from the stacked maps at the four wavelengths, by averaging over one pixel wide rings around the center. The $1 \sigma$ error bars have been estimated with the rms of the pixels along the corresponding rings. The Point Spread Function (PSF), approximated by a Gaussian curve with the FWHM of the IRIS catalogue given 


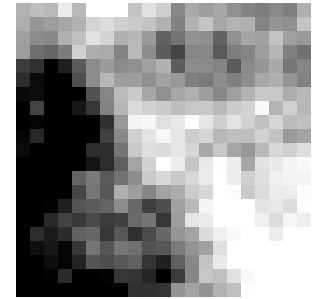

(a) 10

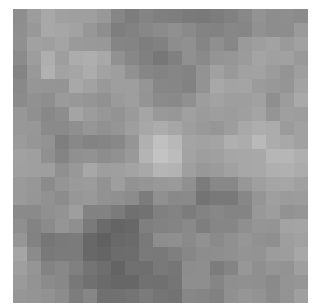

(c) 200

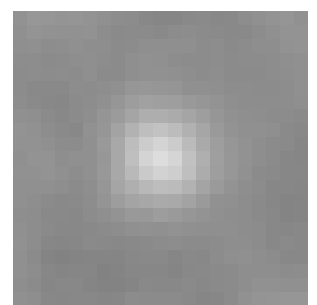

(e) 4000

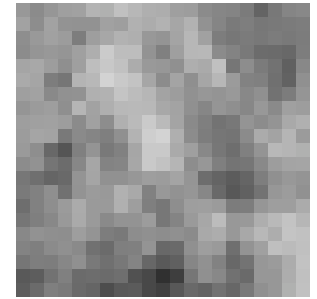

(b) 50

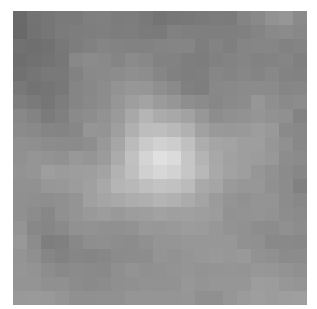

(d) 1000

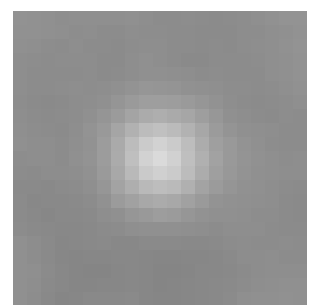

(f) 11507
Fig. 3. Stacked map $\left(31.5^{\prime} \times 31.5^{\prime}\right)$ at $100 \mu \mathrm{m}$ after 10 a), $\left.50 \mathbf{b}\right)$, 200 c), 1000 d), 4000 e), and 11507 f) cluster maps summed. The intensity is scaled between -50000 to $50000 \mathrm{Jy} / \mathrm{sr}$ for all the maps.

in Sect. 2.1, has been overplotted with a dotted line. It first appears that the galaxy clusters are resolved. Neverthless the inaccuracy in the position of the cluster center implies an artificial extension of the profiles. This has been tested in Fig. 6 by plotting the profiles at $100 \mu \mathrm{m}$ obtained for a catalogue of distant clusters (LCDSC dealing with $z \geq 0.3$, Gonzales et al. 2001) and for a catalogue of nearby clusters (WBL dealing with $0.01 \leq z \leq 0.03$, White et al. 1999). The two profiles for nearby (solid line) and distant (dashed line) clusters are very similar (see Fig. 6). Moreover a new PSF, expressed as the product of the resolution given by IRIS and an inaccuracy of $2^{\prime}$ in the position of the cluster, has been overplotted (dotted line), and matches very well the profiles obtained. The fact that the far and nearby clusters have the same extension implies that this extension is an artifact due to the inaccuracy in the position of cluster. The galaxy clusters are not resolved by this method. Moreover this average emission from galaxy clusters is only a low estimate, because of the dilution in the IRAS beam of the smaller clusters, and the subtraction of the mean level of emission for the very nearby clusters extending beyond the $30 \times 30$ arcmin field of view.

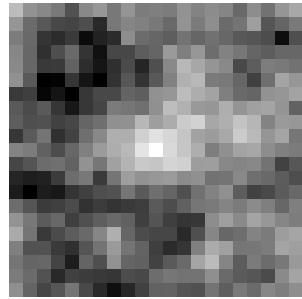

(a) $12 \mu \mathrm{m}$

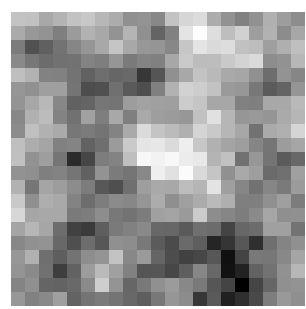

(c) $25 \mu \mathrm{m}$

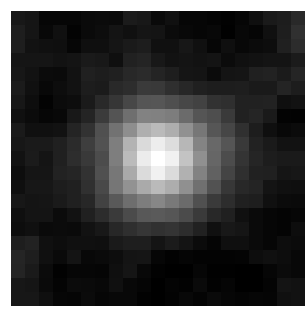

(e) $60 \mu \mathrm{m}$

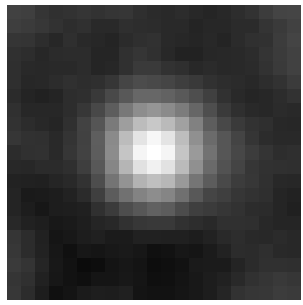

(g) $100 \mu \mathrm{m}$

Stacked maps

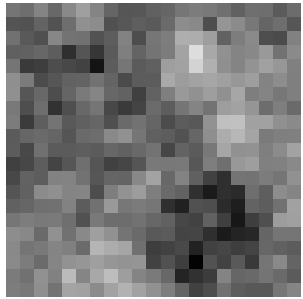

(b) $12 \mu \mathrm{m}$

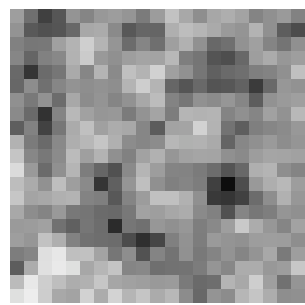

(d) $25 \mu \mathrm{m}$

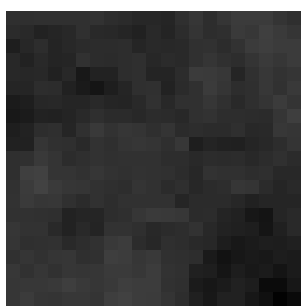

(f) $60 \mu \mathrm{m}$

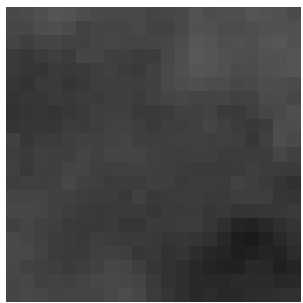

(h) $100 \mu \mathrm{m}$

On/Off maps
Fig. 4. Left panels: stacked maps $\left(31.5^{\prime} \times 31.5^{\prime}\right)$ at the four wavelengths a) $12 \mu \mathrm{m}$ (scaling [-1400; 2400] Jy/sr), c) $25 \mu \mathrm{m}([-2300 ; 2300])$, e) $60 \mu \mathrm{m}([-2300 ; 1500])$ and $\mathbf{g}) 100 \mu \mathrm{m}([-7000 ; 30000])$. Right panels: on/off stacked maps at the same wavelengths with the same greyscale as the corresponding wavelengths.

\subsection{Probing the detection}

The high signal-to-noise ratio of the detection implies a high level of confidence. Neverthless, we have checked that the central peak is not the result of the stacking method itself with the so-called depointing test and On/Off test, and that this mean emission is not only due to the high emission of a few isolated clusters of galaxies with the so-called Bin test.

\subsubsection{The depointing test}

To check that the method does not introduce any artefacts, we apply it on "empty" locations of the sky, without galaxy clusters. Instead of choosing this new set of maps randomly on the 

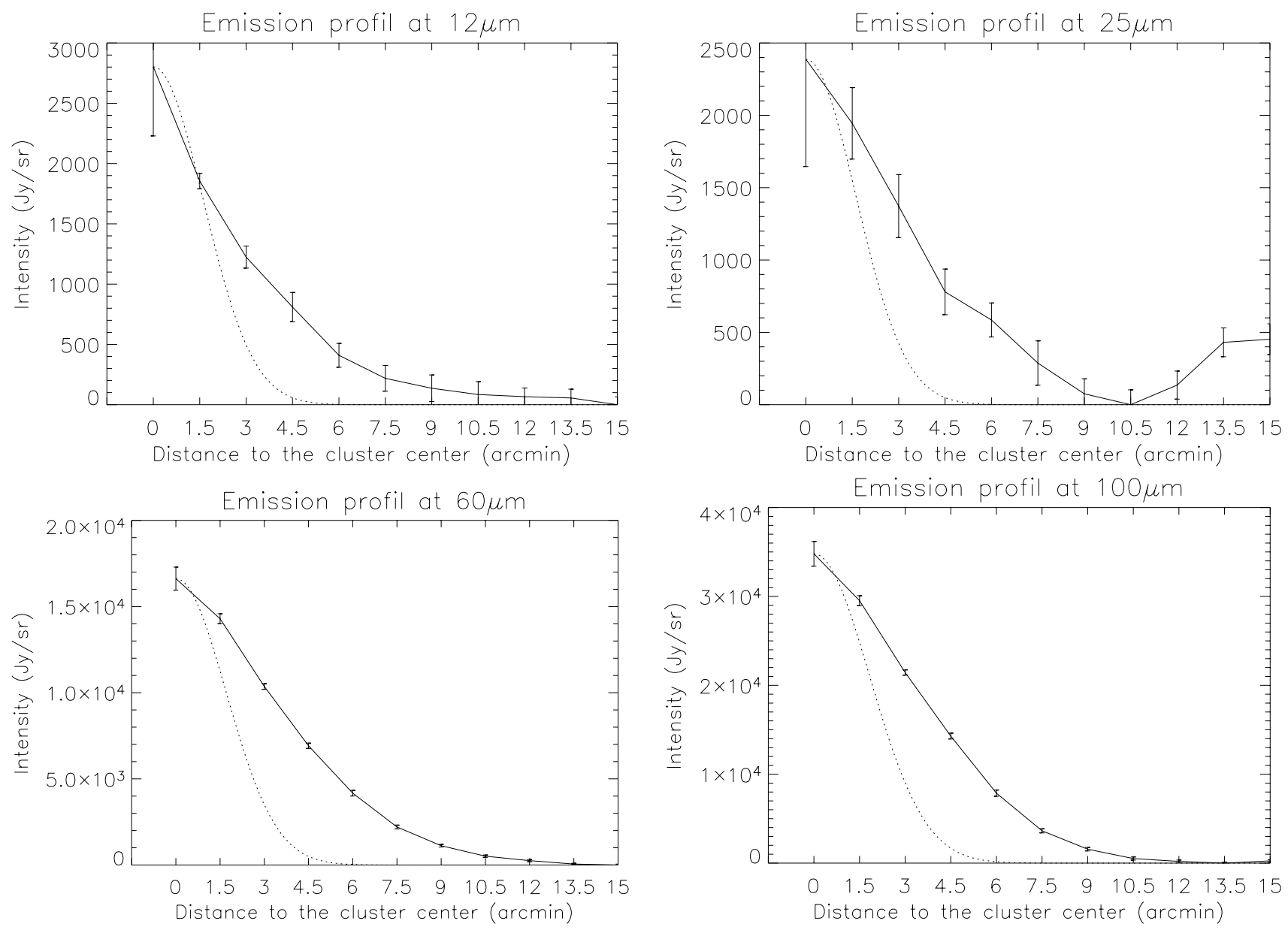

Fig. 5. Intensity profiles (in Jy/sr) of the averaged dust emission from galaxy clusters for the different wavelengths. These values have been obtained by meaning the pixels along circles on the stacked maps. The error bars $(1 \sigma)$ are estimated with the standard deviation of the pixels along the corresponding circles. The profile in dotted line is the PSF.

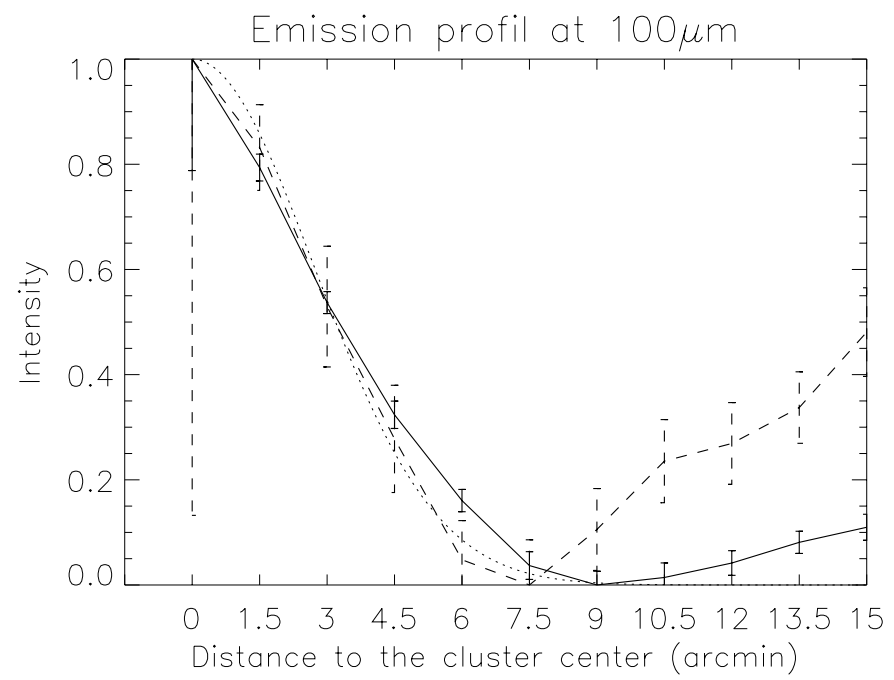

Fig. 6. Intensity profiles (in arbitrary scaling) of the averaged dust emission from galaxy clusters obtained with two different catalogues: WBL (solid line) for nearby clusters and LCDSC (dashed line) for distant clusters. The PSF resulting from the IRIS resolution associated to an error of $2^{\prime}$ in the position of the cluster center has been overplotted (dotted line).
Table 2. Intensity of the central peak and $1 \sigma$ error (defined as the rms of the map outside the central peak) of the averaged IR emission from clusters of galaxies, at each wavelength. $\sigma_{\text {IRIS }}$ represents the upper limit of the noise level of the IRIS maps divided by $\sqrt{11507}$.

\begin{tabular}{cccc}
\hline \hline$\lambda$ & $I_{\lambda}$ & $\sigma$ & $\sigma_{\text {IRIS }}$ \\
\hline$(\mu \mathrm{m})$ & $(\mathrm{Jy} / \mathrm{sr})$ & $(\mathrm{Jy} / \mathrm{sr})$ & $(\mathrm{Jy} / \mathrm{sr})$ \\
\hline 12 & 2685 & 573 & 373 \\
25 & 2127 & 742 & 653 \\
60 & 16253 & 670 & 280 \\
100 & 34011 & 1391 & 746 \\
\hline
\end{tabular}

sky, we depointed our initial cluster maps by 31.5 arcmin, in order to obtain a new set of maps with the same distribution on the sky. This was done for 8 depointings around the initial cluster maps, leading to 8 neighbouring mean maps after the stacking process. As observed in Fig. 7 for the $100 \mu$ m maps, no signal has been detected in the neighbouring maps, whereas the dust emission is present in the central maps, centered on galaxy clusters. This confirms the robustness of the stacking method and the validity of the detection of the average dust emission from clusters of galaxies. 

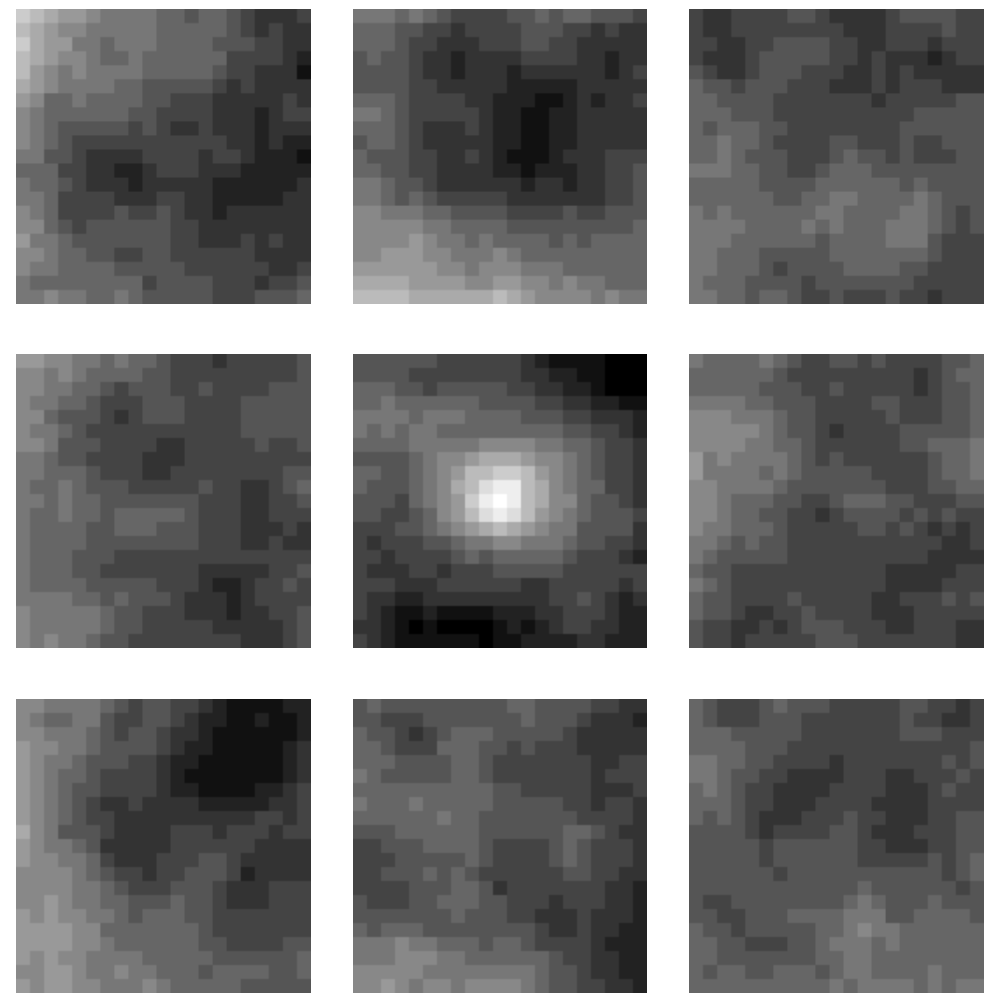

Fig. 7. Depointing Test: the map $\left(31.5^{\prime} \times 31.5^{\prime}\right)$ at the center is the result of the stacking process applied on the cluster catalogue at $100 \mu \mathrm{m}$, whereas the neighbouring maps have been obtained after a depointing of $31.5^{\prime}$ from the initial reference. The grey-scale is the following: from -20000 to $40000 \mathrm{Jy} / \mathrm{sr}$.

Table 3. Intensity of the central peak averaged over the bins and $1 \sigma$ error (defined as the rms over the bins) of the average IR emission from clusters of galaxies, at each wavelength, for the two sets of 10 and 20 bins.

\begin{tabular}{ccc}
\hline \hline$\lambda$ & 10 bins & 20 bins \\
\hline$(\mu \mathrm{m})$ & $(\mathrm{Jy} / \mathrm{sr})$ & $(\mathrm{Jy} / \mathrm{sr})$ \\
\hline 12 & $2712 \pm 260$ & $2638 \pm 519$ \\
25 & $2198 \pm 496$ & $2150 \pm 608$ \\
60 & $16315 \pm 288$ & $16219 \pm 389$ \\
100 & $34138 \pm 798$ & $33916 \pm 945$ \\
\hline
\end{tabular}

\subsubsection{The on/off test}

This second test consists of checking that the signal is not due to a systematic effect. We perform a modified stacking process by alternatively summing and subtracting the cluster maps instead of summing them. The disappearance of the central peak in the resulting averaged maps means that all the cluster maps have almost the same contribution to the final central peak and that this detection is not biased by only a few cluster maps of the sample.

The resulting on/off maps are displayed on the right pannels of Fig. 4, with the same grey-scale as for the mean maps obtained with the classical stacking method (left pannels). By comparing these two sets of maps, we can easily check that the central peaks are not present any more on the on/off maps, while they are obvious on the classical stacked maps.
This central peak can then be considered as a statistical signal and not a spurious emission of galaxy clusters.

\subsubsection{The bin test}

Because of the undetectability of the dust emission of a single galaxy cluster, we cannot derive the standard deviation associated with this average IR intensity detected. In order to estimate it, the total sample of the galaxy clusters was divided into 10 bins, randomly distributed. The entire stacking process was repeated separately on each bin. The size of the bins (about 1400 galaxy clusters) was chosen to reach a sufficient signal-tonoise ratio in the $60 \mu \mathrm{m}$ and $100 \mu \mathrm{m}$ bands to remain confident of the results for each bin. We could then derive an estimate of the standard deviation of the average over the bins. We can observe in Fig. 8 for the $60 \mu \mathrm{m}$ and $100 \mu \mathrm{m}$, that the estimate of the dust emission averaged over the bins (dashed line, see also Table 3 ) is very close to the result obtained for the sample as a whole (dotted line, see also Table 2). The errors derived from the standard deviation over the bins are in very good agreement with the error bars obtained with the global method. The same process was performed with 20 bins (see Fig. 9). The number of cluster maps in each bin is about 700, and a similar intensity of the central peak is observed (see Table 3 ). This rules out the hypothesis of an average intensity only due to a few luminous objects, instead of a contribution of all the galaxy clusters of the sample.

Using the same method: the whole sample was divided into the 10 larger sub-catalogues. The resulting intensity at $60 \mu \mathrm{m}$ 

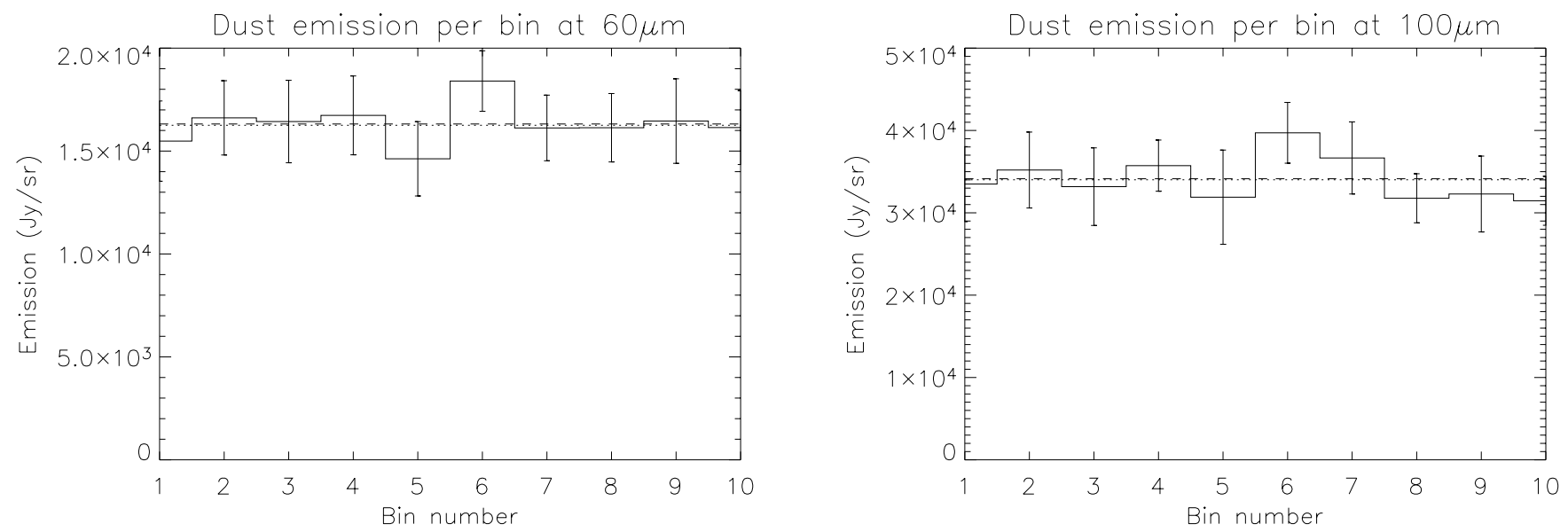

Fig. 8. Bin Test: intensity of the central peak (in Jy/sr) at $60 \mu \mathrm{m}$ (left panel) and $100 \mu \mathrm{m}$ (right panel) for the 10 bins randomly extracted from the whole sample. The average (dashed line) and the error $(1 \sigma)$ over the bins are $16315 \pm 288 \mathrm{Jy} / \mathrm{sr}$ and $34138 \pm 798 \mathrm{Jy} / \mathrm{sr}$ at $60 \mu \mathrm{m}$ and $100 \mu \mathrm{m}$ respectively. The intensity of the central peak obtained for the same sample considered as a whole is overplotted in dotted line. The errors bars represent the $1 \sigma$ error estimated for each bin (rms of the summed map outside the peak).
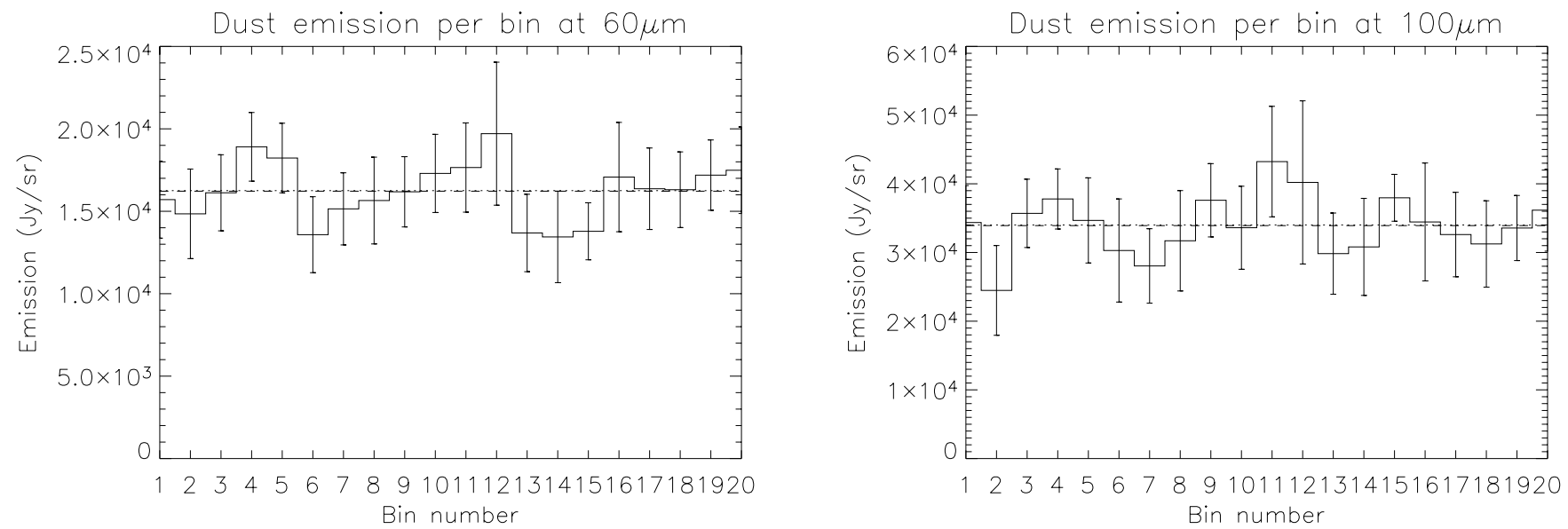

Fig. 9. Bin Test: same as Fig. 8 with 20 bins. The average (dashed line) and the standard deviation over the bins are $16219 \pm 389 \mathrm{Jy} / \mathrm{sr}$ and $33916 \pm 945 \mathrm{Jy} / \mathrm{sr}$ at $60 \mu \mathrm{m}$ and $100 \mu \mathrm{m}$ respectively.
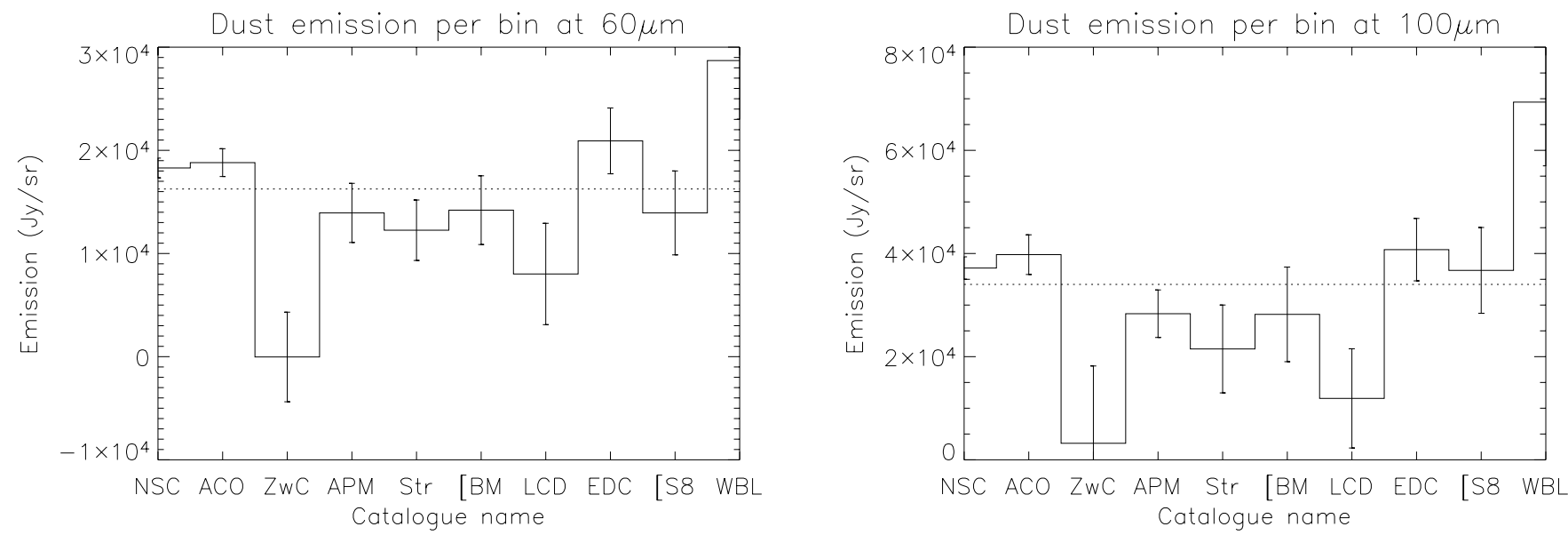

Fig. 10. Bin Test Catalogue: intensity of the central peak (in Jy/sr) and $1 \sigma$ error bars at $60 \mu \mathrm{m}$ (left panel) and $100 \mu \mathrm{m}$ (right panel) for the 10 bigger sub-catalogues. The intensity of the central peak obtained for the whole sample is overplotted with a dotted line. 
and $100 \mu \mathrm{m}$ is shown for each catalogue in Fig. 10. Except 3 catalogues (detailed below) that show special features, the others follow the averaged results presented in Sect. 3.2. The Zwicky catalogue (Zwicky et al. 1961, called "ZwC" in Fig. 10) shows no detection either at $60 \mu \mathrm{m}$ or $100 \mu \mathrm{m}$ : this can be explained by the nature of this catalogue that contains many very diffuse objects. The LCDSC (Gonzales et al. 2001, called "LCD" in Fig. 10) is a deep survey, selecting clusters with high redshift $(z \geq 0.3)$ : due to their large distance and small angular extension, these objects are less luminous and diluted in the IRAS beam, explaining the smaller intensity detected. On the contrary, the WBL (White et al. 1999) has about two times the averaged emission obtained globaly. This can be explained by the fact that this catalogue contains very nearby clusters of galaxies, that are often associated with X-ray bright ICM.

Using these three tests, the depointing test, On/Off test, and bin test, the detection of the dust emission from clusters of galaxies has been confirmed with success. Firstly this emission is not an artefact due to the stacking method itself. Secondly the resulting intensity is the averaged emission from galaxy clusters and cannot be attributed to a small fraction of clusters. For these reasons, we are confident of our detection.

\section{Conclusion}

We have implemented a statistical method to detect the IR emission from galaxy clusters. This method computes the average of a high number of small maps centered on galaxy clusters, by taking into account the local point sources and the discrepancy between maps. Because of the random distribution of the IR background, the central peak can be revealed progressively. This method has been applied to a large catalogue of 14588 references of clusters of galaxies. We have found evidence for central IR emission at the four IRAS wavelengths 12 , 25, 60 and $100 \mu \mathrm{m}$, with a high level of confidence, except for $25 \mu \mathrm{m}$ where the signal-to-noise ratio is only 2.75 . These detections have been tested and validated independently with three different procedures. The results are in a good agreement with the previous observations of Kelly \& Rieke (1990) at $100 \mu \mathrm{m}$, but are lower by a factor of 2 at $60 \mu \mathrm{m}$. The bias of selection of the cluster sample could explain such a difference.

Of the 4 IRAS bands measured, the signal is strongest in the $100 \mu \mathrm{m}$ band with an intensity of $34011 \mathrm{Jy} / \mathrm{sr}$, i.e. only a few hundredths of the background level, that may typically be 5-10 MJy/sr at $100 \mu \mathrm{m}$ (averaged over the sky). We have not determined the origin of this slight IR emission. The counterpart at IR wavelengths of the cluster free-free emission due to the hot intracluster gas could be one of the candidates. The freefree brightness temperature of an optically thin and hot plasma of ionized hydrogen is given by (Osterbrock 1989):

$T_{\mathrm{b}}=\frac{5.43}{v_{10}^{2} T_{4}^{1 / 2}} g_{\mathrm{ff}} E M$

where the brightness temperature in given in $\mu \mathrm{K}, v_{10}$ is the wavelength in $10 \mathrm{GHz}, T_{4}$ is the temperature of the gas in $10^{4} \mathrm{~K}, g_{\mathrm{ff}}$ is the Gaunt factor $\left(g_{\mathrm{ff}} \approx 1\right.$, Nozawa et al. 1998 ), and $E M$ is the emission measure in $\mathrm{pc} \mathrm{cm}^{-6}$ given by $E=\int n_{\mathrm{e}}^{2} \mathrm{~d} l$ with $n_{\mathrm{e}}$ the electron density. We chose typical properties of rich galaxy clusters: $T=10^{7} \mathrm{~K}, n_{\mathrm{e}}=10^{-3} \mathrm{~cm}^{-3}$, and a core radii of $R_{\mathrm{c}}=0.5 \mathrm{Mpc}$. With these characteristics, the emission measure integrated above two core radius yields $E \approx 1$. At $100 \mu \mathrm{m}(v \approx 3000 \mathrm{GHz})$, the free-free brightness intensity is then given by $I_{\mathrm{b}} \approx 5 \mathrm{Jy} / \mathrm{sr}$, that is much lower than the average IR emission detected in the galaxy clusters. Moreover the free-free emission has a very flat spectrum, whereas the ratio between the galaxy cluster intensity at $100 \mu \mathrm{m}$ and $12 \mu \mathrm{m}$ is above 13. For these reasons, the bremsstrahlung cannot be responsible for the IR emission observed at the center of galaxy clusters.

Dust remains the best candidate. The key question now is wether this dust is really intergalactic or located inside the galaxies of the clusters. According to Popescu et al. (2000) the intensity of the emission from the intergalactic dust component could be up to $20000 \mathrm{Jy} / \mathrm{sr}$ at $100 \mu \mathrm{m}$ (of which about $10000 \mathrm{Jy} / \mathrm{sr}$ is from dust produced by intergalactic stars and up to $10000 \mathrm{Jy} / \mathrm{sr}$ is from dust accreted onto the cluster). To what extent is our detection produced by intergalactic dust? This crucial question will be discussed extensively in Paper II.

Acknowledgements. We are indebted to the referee C. Popescu for his detailed reading that lead to a considerable improvement for the paper. We are also grateful to M.-A. Miville-Deschênes and G. Lagache for the private communication of the IRIS data before publication.

\section{References}

Abell, G. O., Corwin, H. G., \& Olowin, R. P. 1989, ApJS, 70, 1 Aguirre, A., Hernquist, L., Katz, N., Gardner, J., \& Weinberg, D. 2001a, ApJ, 556, L11

Aguirre, A., Hernquist, L., Schaye, J., et al. 2001b, ApJ, 561, 521

Aguirre, A., Hernquist, L., Schaye, J., et al. 2001c, ApJ, 560, 599

Bahcall, N. A., McKay, T., Annis, J., et al. 2003, ApJS, 148, 243

Beichman, C. A., Neugebauer, G., Habing, H. J., Clegg, P. E., \& Chester, T. J. 1988, NASA RP-1190, Vol. 1, 0, IRAS Catalogs and Atlases: Explanatory Supplement

Bogart, R. S., \& Wagoner, R. V. 1973, ApJ, 181, 609

Boyle, B. J., Fong, R., \& Shanks, T. 1988, MNRAS, 231, 897

Bregman, J. N., McNamara, B. R., \& O’Connell, R. W. 1990, ApJ, 351,406

Cen, R., \& Ostriker, J. P. 1999, ApJ, 519, L109

Cox, C. V., Bregman, J. N., \& Schombert, J. M. 1995, ApJS, 99, 405

Dalton, G. B., Maddox, S. J., Sutherland, W. J., \& Efstathiou, G. 1997, MNRAS, 289, 263

Duc, P.-A., Braine, J., Lisenfeld, U., Amram, P., \& Brinks, E. 2002, Ap\&SS, 281, 347

Durrell, P. R., Ciardullo, R., Feldmeier, J. J., Jacoby, G. H., \& Sigurdsson, S. 2002, ApJ, 570, 119

Duus, A., \& Newell, B. 1977, ApJS, 35, 209

Dwek, E. 1987, ApJ, 322, 812

Feldmeier, J. J., Ciardullo, R., \& Jacoby, G. H. 1998, ApJ, 503, 109

Ferguson, H. C., Tanvir, N. R., \& von Hippel, T. 1998, Nature, 391, 461

Gal, R. R., de Carvalho, R. R., Lopes, P. A. A., et al. 2003, AJ, 125, 2064

Gal-Yam, A., Maoz, D., Guhathakurta, P., \& Filippenko, A. V. 2003, AJ, 125, 1087

Gautier, T. N., Boulanger, F., Perault, M., \& Puget, J. L. 1992, AJ, 103, 1313

Gerhard, O., Arnaboldi, M., Freeman, K. C., \& Okamura, S. 2002, ApJ, 580, L121

Gnedin, N. Y., \& Ostriker, J. P. 1997, ApJ, 486, 581 
Gonzalez, A. H., Zaritsky, D., Dalcanton, J. J., \& Nelson, A. 2001, ApJS, 137, 117

Guiderdoni, B., Bouchet, F. R., Puget, J., Lagache, G., \& Hivon, E. 1997, Nature, 390, 257

Gunn, J. E., Hoessel, J. G., \& Oke, J. B. 1986, ApJ, 306, 30

Helou, G., \& Beichman, C. A. 1990, From Ground-Based to SpaceBorne Sub-mm Astronomy, 117

Hu, E. M. 1992, ApJ, 391, 608

Karachentsev, I. D., \& Lipovetskii, V. A. 1969, Soviet Astron., 12, 909

Kelly, D. M., \& Rieke, G. H. 1990, ApJ, 361, 354

Kiss, C., Ábrahám, P., Klaas, U., Juvela, M., \& Lemke, D. 2001, A\&A, 379,1161

Lumsden, S. L., Nichol, R. C., Collins, C. A., \& Guzzo, L. 1992, MNRAS, 258, 1

Miville-Deschênes, M.-A., \& Lagache, G. 2004, ApJS, in press [arXiv:astro-ph/0412216]

Montier, L. A., \& Giard, M. 2004, A\&A, 417, 401

Nozawa, S., Itoh, N., \& Kohyama, Y. 1998, ApJ, 507, 530

Osterbrock, D. E. 1989, Research supported by the University of California, John Simon Guggenheim Memorial Foundation, University of Minnesota, Mill Valley, CA, University Science Books, 422
Popescu, C. C., Tuffs, R. J., Fischera, J., \& Völk, H. 2000, A\&A, 354, 480

Postman, M., Lauer, T. R., Oegerle, W., \& Donahue, M. 2002, ApJ, 579, 93

Quillen, A. C., Rieke, G. H., Rieke, M. J., Caldwell, N., \& Engelbracht, C. W. 1999, ApJ, 518, 632

Romani, R. W., \& Maoz, D. 1992, ApJ, 386, 36

Scodeggio, M., Olsen, L. F., da Costa, L., et al. 1999, A\&AS, 137, 83

Shectman, S. A. 1985, ApJS, 57, 77

Stickel, M., Lemke, D., Mattila, K., Haikala, L. K., \& Haas, M. 1998, A\&A, 329, 55

Stickel, M., Klaas, U., Lemke, D., \& Mattila, K. 2002, A\&A, 383, 367

Theuns, T., \& Warren, S. J. 1997, MNRAS, 284, L11

Wheelock, S. L., et al. 1994, NASA STI/Recon Technical Report N, 95, 22539

White, R. A., Bliton, M., Bhavsar, S. P., et al. 1999, AJ, 118, 2014

Wise, M. W., O'Connell, R. W., Bregman, J. N., \& Roberts, M. S. 1993, ApJ, 405, 94

Zwicky, F. 1957, PASP, 69, 518

Zwicky, F., et al. 1961, California Inst. Techn., 1, 0 